\title{
Microbiote et os
}

Lidia Ibáñez ${ }^{a, b, c}$, Matthieu Rouleau ${ }^{a, b}$, Abdelilah Wakkachª,b, Claudine Blin-Wakkacha,b

a CNRS UMR7370, LP2M, Faculté de Médecine, 06107 Nice, France

b Université Nice Sophia Antipolis, 06100 Nice, France

c Department of Pharmacy, Cardenal Herrera-CEU University, 46115 Alfara del Patriarca, València, Spain

Auteur correspondant: Claudine Blin-Wakkach, CNRS UMR7370, LP2M, Faculté de Médecine, 28 avenue de Valombrose, 06107 Nice cedex 2, France.

E-mail: blin@unice.fr 


\section{Résumé}

Le microbiote est aujourd'hui considéré comme un tissu qui interagit de façon réciproque avec les systèmes digestif, immunitaire, endocrinien et nerveux. II affecte donc les réponses cellulaires de nombreux organes et est impliqué dans un nombre croissant de processus physiopathologiques souvent liés à une réponse inflammatoire. En particulier, les 10 dernières années ont été marquées par la mise en évidence de l'influence du microbiote sur la régulation de la masse osseuse et le développement de pathologies osseuses telles qu'ostéoporose ou rhumatismes inflammatoires associés à une diminution de la densité osseuse. Ainsi, les souris dépourvues de microbiote présentent des modifications de la masse osseuse qui sont corrigées après recolonisation de leur microbiote. Les souris qui développent une ostéoporose associée à une déficience en œstrogènes présentent également des variations de la composition du microbiote, un effet également décrit dans quelques études chez l'homme. L'administration de probiotiques réduit la perte osseuse chez les animaux déficients en œstrogènes. L'effet du microbiote sur l'os implique des mécanismes complexes incluant entre autres la modulation de l'activation des lymphocytes $T$ $\mathrm{CD}^{+}$, le contrôle de la production de cytokines ostéoclastogéniques ainsi que des changements hormonaux. Cette complexité participe à la discordance observée entre certaines études dont les résultats varient en fonction de l'âge, du sexe, de l'environnement génétique et de la durée des traitements. Une meilleure compréhension de ces mécanismes est donc encore nécessaire mais ces résultats ouvrent des perspectives intéressantes pour les pathologies osseuses basées sur la manipulation du microbiote.

\section{Mots clés $=$}

Ostéoclastes, ostéoporose, ostéoimmunologie, lymphocytes T, microbiote 


\section{1- Introduction}

Composé de 100000 milliards de micro-organismes, le microbiote entretient un dialogue dynamique et permanent avec les cellules de l'hôte [1]. II est aujourd'hui considéré comme un organe participant aux fonctions digestives. II transforme des composants alimentaires complexes tels que fibres et carbohydrates en métabolites assimilables par l'hôte comme les acides gras à chaînes courtes [2]. Grâce aux composants qu'elles produisent, les bactéries commensales modulent les réponses cellulaires de l'hôte, et participent au maintien de l'intégrité de la barrière intestinale, à la protection contre les agents pathogènes et au développement et à la régulation du système immunitaire. Ceci est particulièrement important pour le système immunitaire de la muqueuse intestinale dont la fonction est de maintenir une tolérance vis-à-vis des antigènes alimentaires et du microbiote commensal tout en protégeant des microorganismes pathogènes. Ainsi, le microbiote régule le développement et la fonction des cellules lymphoïdes et la polarisation des lymphocytes $T$ dans l'intestin, en particulier les lymphocytes Th17, et la production de cytokines [3,4].

La composition du microbiote peut varier au cours de la vie en fonction de l'âge, de facteurs génétiques, du régime alimentaire, de la prise médicamenteuse ou du statut immunitaire de l'hôte. Ses interactions avec l'hôte peuvent être altérées par les variations de la composition, de la diversité et de la fonction bactérienne selon un processus appelé dysbiose. Le microbiote ne joue alors plus son rôle protecteur, la barrière intestinale est altérée et l'hôte ne contrôle plus efficacement la dissémination des composants du microbiote dans les tissus. Cela résulte en une stimulation du système immunitaire pouvant conduire à de nombreuses pathologies. Une dysbiose est ainsi associée aux pathologies du système intestinal telles que la maladie de Crohn, le syndrome du côlon irritable, la maladie coeliaque, mais également à des pathologies systémiques telles que les maladies métaboliques, cardiovasculaires, neurodégénératives et les rhumatismes inflammatoires [57]. Au cours des dernières années, l'impact du microbiote sur le tissu osseux a été évalué dans des modèles animaux dépourvus de microbiote (axéniques) ou chez lesquels il est modifié par l'administration d'antibiotiques ou de probiotiques, et également chez l'homme. Malgré certaines divergences entre ces études, le microbiote apparaît aujourd'hui comme un régulateur majeur de la densité osseuse principalement grâce à ses effets sur le système immunitaire.

\section{2- Interactions ostéo-immunologiques et résorption osseuse}

Le système immunitaire joue un rôle essentiel dans le contrôle de la densité osseuse en condition pathologique. RANKL (Receptor Activator of NFKB (RANK) Ligand), la principale cytokine impliquée dans la différenciation des ostéoclastes (OCLs), est produite par les cellules mésenchymateuses, les ostéoblastes (OBLs) et les ostéocytes de la moelle 
osseuse, et en conditions inflammatoires elle est également produite par les lymphocytes $T$ $\mathrm{CD}^{+}$activés [8,9]. Ces lymphocytes produisent aussi d'autres cytokines telles que l'interleukine (IL)-17 et le TNF $\alpha$ (tumor necrosis factor) qui stimulent également l'ostéoclastogenèse [10,11]. La modification du niveau d'activation des lymphocytes $T$ impacte donc la différenciation ostéoclastique $[12,13]$. Parmi les différentes sous-populations de lymphocytes T CD4+ auxiliaires, seules les cellules Th17 ont été décrites comme proostéoclastogéniques in vitro [14]. In vivo, les cellules Th17 sont associées à une augmentation de la différenciation des OCLs chez la souris et chez l'homme en conditions inflammatoires $[13,15,16]$. Cette interaction entre cellules Th17 et OCLs a également été mise en évidence dans le contexte de la maladie de Crohn chez la souris et chez l'homme $[16,17]$. Les cellules Th17 activées dans l'intestin enflammé produisent des taux élevés de facteurs ostéoclastogéniques RANKL, IL-17 et TNF $\alpha$ [17]. Elles migrent dans la moelle osseuse où elles augmentent considérablement la différenciation des OCLs via leur production de cytokines et la stimulation de l'expression de RANKL par les cellules mésenchymateuses [17]. Elles stimulent également dans les cellules mésenchymateuses l'expression de chimiokines (MCP1, MIP1 $\alpha$ ) attirant les précurseurs monocytaires des OCLs ce qui augmente le recrutement de ces précurseurs dans la moelle osseuse [17]. Ces cellules Th17 sont également présentes dans le sang de patients atteints de maladie de Crohn, avec les mêmes propriétés ostéoclastogéniques et participent donc probablement à la diminution de densité osseuse très fréquemment observée dans cette maladie [16,17]. Les cellules Th17 activées au niveau du tractus digestif sont donc des acteurs majeurs de la destruction osseuse inflammatoire.

De façon intéressante, les cellules Th17 sont absentes dans le tissu intestinal chez les souris dépourvues de microbiote et leur différenciation est induite très efficacement par certaines espèces bactériennes ce qui démontre l'importance du microbiote dans leur émergence [3,4]. Les cellules Th17 ont une grande plasticité phénotypique et fonctionnelle [18]. Elles jouent un rôle protecteur vis à vis des bactéries pathogènes [19] mais elles sont aussi impliquées dans de nombreuses maladies inflammatoires chroniques y compris celles associées avec une destruction osseuse telles que la maladie de Crohn [20], l'arthrite [21], la spondyloarthrite [22], ou le psoriasis [23], entre autres. Ces données soutiennent donc l'hypothèse d'un lien entre microbiote intestinal et résorption osseuse via l'émergence des cellules Th17.

\section{3- Microbiote et développement osseux}

Au début des années 2000, plusieurs études cliniques ont suggéré une association entre une prolifération excessive des bactéries intestinales et une diminution de la densité minérale osseuse dans le syndrome de surcroissance bactérienne de l'intestin grêle [24]. 
Les patients présentent une masse osseuse faible, une ostéomalacie et pour certains, des niveaux élevés des cytokines inflammatoires TNF $\alpha$ et d'IL-1 et une augmentation de l'activation des OCLs. Mais ce syndrome est également caractérisé par un déficit nutritionnel dû à la consommation des nutriments par les bactéries au détriment de l'hôte et une malabsorption intestinale de calcium, de phosphate et de vitamine $\mathrm{D}$ qui participent aux conséquences osseuses de la pathologie [24]. Ces observations ne permettent donc pas d'établir un lien direct entre microbiote et phénotype osseux.

Ce n'est que 10 ans plus tard que l'implication du microbiote intestinal dans le développement osseux a été démontrée grâce à l'étude de souris dépourvues de microbiote. Étant donné l'impact du microbiote sur le métabolisme, son absence dès la naissance induit de nombreux changements physiologiques et métaboliques tels qu'une réduction de l'apport énergétique et de l'absorption de certains nutriments et vitamines, une diminution de la taille et du poids corporel et de nombreux organes [25]. Elle entraine également une immaturité des systèmes immunitaire, vasculaire, endocrinien, intestinal et nerveux qui sont tous impliqués dans la régulation de la masse osseuse (Figure 1) [26,27].

L'absence de microbiote dès la naissance conduit chez les souris femelles C57BI/6J à une densité minérale osseuse plus élevée au niveau trabéculaire et cortical que chez les animaux en élevage conventionnel [28]. Cette augmentation de densité osseuse est liée à une diminution du nombre de précurseurs des OCLs, du nombre d'OCLs matures et donc de la résorption osseuse, mais pas à une modification de la formation osseuse. Elle est associée à une diminution de la fréquence des lymphocytes $\mathrm{T} \mathrm{CD4}^{+}$et à une baisse de l'expression de TNF $\alpha$ et d'IL-6 dans la moelle osseuse qui participent à la réduction de la résorption osseuse [28]. La colonisation des souris axéniques avec le microbiote de souris en élevage conventionnel normalise la densité osseuse et les paramètres immunitaires confirmant que le microbiote contrôle la différenciation des OCLs au cours de la croissance du squelette [28]. Parmi les différents récepteurs permettant au système immunitaire de reconnaître les bactéries, les récepteurs NOD1 et NOD2 (Nucleotide-binding Oligomerization Domain proteins) qui lient les peptidoglycanes bactériens et activent la voie $\mathrm{NF} \kappa \mathrm{B}$, jouent un rôle déterminant dans les effets du microbiote sur l'os. En effet, ni l'expression de TNF $\alpha$ et RANKL ni la densité osseuse ne sont affectées par les variations de microbiote chez les souris invalidées pour ces deux gènes [29].

Cependant, l'effet du microbiote sur le développement osseux est plus complexe qu'il n'apparaît. En effet, une autre étude réalisée en 2016 a rapporté des résultats inverses chez des souris axéniques mâles juvéniles Balb/c. Ces animaux présentent une diminution des principaux paramètres de croissance osseuse et des fémurs plus courts que les contrôles en élevage conventionnel [30]. La divergence de résultats entre les deux études peut 
s'expliquer par des différences dans le fond génétique, le sexe ou l'âge des animaux. Les souches $\mathrm{C} 57 \mathrm{BI} / 6 \mathrm{~J}$ et Balb/C diffèrent par la diversité de leur microbiote, leur taux d'immunoglobines A (IgAs) qui modulent cette diversité et leur profil lymphocytaire T [31,32]. La durée des traitements est également un paramètre extrêmement important. La colonisation de souris axéniques par le microbiote de souris normales conduit à une augmentation à la fois de la résorption et de la formation osseuse. Mais l'augmentation de la résorption n'est que transitoire dans les quatre premières semaines après cette colonisation, révélant un effet catabolique aigu du microbiote sur l'os [33]. À plus long terme, c'est la formation osseuse qui devient prédominante entraînant une augmentation de la croissance osseuse et une normalisation de la masse osseuse. Cela démontre un effet anabolique à long terme du microbiote sur l'os [33]. Cet effet du microbiote a été associé à une augmentation de la production d'Insuline Growth Factor (IGF)-1. Les taux d'IGF-1 dans le sérum et la moelle osseuse sont bas chez les souris axéniques ou traitées aux antibiotiques. Ils augmentent après recolonisation du microbiote des animaux ou après un régime riche en acides gras à chaînes courtes qui sont produits par la dégradation des polysaccharides par le microbiote [33]. Étant donné le rôle majeur de l'IGF1 dans le développement du squelette [34], le contrôle de sa production par le microbiote est un des mécanismes pouvant expliquer l'effet du microbiote sur la croissance osseuse.

Un autre facteur important est la différence liée au sexe. Cette hypothèse est confortée par le phénotype différent observé entre souris C57BI/6J mâles et femelles traitées dès la naissance par de faibles doses de pénicilline qui entraînent des altérations significatives du microbiote [35]. La densité minérale osseuse augmente à l'âge adulte par rapport aux animaux non traités mais uniquement chez les femelles [35]. De plus, l'augmentation de densité osseuse observée après recolonisation de souris axéniques est plus importante à long terme chez les mâles que chez les femelles [33]. De façon intéressante, il a été démontré chez la souris que le microbiote est équivalent avant la puberté mais diffère à l'âge adulte entre mâles et femelles et ces différences participent à une susceptibilité accrue à certaines pathologies chroniques comme le diabète de type 1 chez les femelles [36]. Une différence de microbiote liée au sexe a également été observée chez l'Homme $[37,38]$. Ces observations démontrent donc que les interactions réciproques entre microbiote et hormones sexuelles influencent la composition du microbiote et la réponse de l'hôte vis-à-vis du microbiote, en particulier au niveau osseux [39].

\section{4- Microbiote et destruction osseuse}

L'influence du microbiote sur la masse osseuse a donc logiquement été évaluée dans le contexte de l'ostéoporose due à la déficience en œstrogènes. La baisse de la production d'œstrogènes à la ménopause conduit à une réduction de la densité osseuse à la fois par la 
diminution de la formation osseuse et par l'augmentation de sa dégradation. Dans le modèle murin d'ostéoporose induit par ovariectomie chez la souris, la stimulation de l'activité des OCLs est liée à une augmentation de production de RANKL et de TNF $\alpha$ dans la moelle osseuse [40]. Cette production est médiée par les lymphocytes T CD4+ [40]. Cette régulation est confirmée par le fait que les souris déficientes en lymphocytes T CD4 ${ }^{+}$sont résistantes à la perte osseuse après ovariectomie [41]. Ces résultats ont été vérifiés chez des patientes atteintes d'ostéoporose post-ménopausique : par rapport aux femmes en pré-ménopause ou post-ménopause sans ostéoporose, les patientes ménopausées avec ostéoporose ont une production plus élevée de RANKL et TNF $\alpha$ par les lymphocytes T CD4+ circulants [42].

Étant donné l'importance du microbiote dans le contrôle de l'activation de lymphocytaire [4] et ses interactions réciproques avec les hormones sexuelles [36], son impact sur la baisse de densité osseuse induite par la diminution du taux d'estrogènes a été évalué chez la souris. Plusieurs études ont montré un effet protecteur de probiotiques tels que Lactobacillus (L) reuteri, L. paracasei, L. plantarum, Bifidobacterium (B) longum, ou de mélange de différentes souches (B. breve, B. longum, B. infantis, $L$. acidophilus, $L$. plantarum, L. paracasei, L. bulgaricus, et Streptococcus thermophilus) sur la perte osseuse induite par ovariectomie chez la souris [43-45]. Ces résultats ont été confirmés dans un modèle d'ostéoporose induit par traitement par le leuprolide, un agoniste de la $\mathrm{GnRH}$ (Gonadotropin Releasing Hormone) qui conduit à long terme à un blocage de la production d'hormones sexuelles et en particulier à une déficience en œstrogènes. Seules les souris en élevage conventionnel présentent une diminution de la masse osseuse liée à cette déficience en œstrogènes, mais pas les souris axéniques [46]. Chez les souris en élevage conventionnel, cette déficience en œstrogènes augmente la perméabilité intestinale, augmente la proportion de lymphocytes T CD4+ produisant du RANKL, de I'IL-17 et du TNF $\alpha$ ce qui conduit à une ostéoclastogenèse plus élevée (figure 2) [46]. Au contraire, les souris sans microbiote sont résistantes à toutes ces modifications [46]. Une étude préliminaire réalisée chez un petit nombre de patients (6 par groupe) a révélé des modifications du microbiote entre témoins sains, patients ostéopéniques et patients ostéoporotiques. Cette observation doit être confirmée chez un nombre plus important de patients. Mais elle suggère que le lien entre dysbiose et ostéoporose peut également être impliqué dans l'ostéoporose chez l'homme [47].

Au-delà de l'ostéoporose, les modifications du microbiote ont un rôle majeur dans les rhumatismes inflammatoires dans lesquels une destruction osseuse est observée. Chez la souris ou le rat, l'absence de microbiote ou sa modulation par des antibiotiques rend les animaux moins sensibles à l'arthrite et la spondyloarthrite $[48,21]$ et chez l'homme, une dysbiose intestinale est observée chez les patients atteints de polyarthrite rhumatoïde ou de spondyloarthrite [49-51]. De plus, l'administration de probiotiques protège de l'inflammation 
et de la perte osseuse chez les animaux arthritiques [52]. L'influence du microbiote sur l'os n'est donc pas limitée aux pathologies impliquant des modifications hormonales et peut impliquer des mécanismes très variés.

Les données récentes de la littérature révèlent qu'ostéoporose et rhumatismes inflammatoires partagent une composante immunitaire commune. La barrière intestinale est essentielle aux interactions hôte - microbiote et son intégrité est altérée en condition de dysbiose favorisant la dissémination des bactéries et des facteurs qu'elles produisent. Une telle altération est observée dans les rhumatismes inflammatoires comme dans la déficience en œstrogènes [46,53,54]. Dans les deux cas, cette altération est associée à une augmentation de l'activation des lymphocytes $\mathrm{T} \mathrm{CD}^{+}$et de la production des cytokines inflammatoires et ostéoclastogéniques IL-17, TNF $\alpha$, IL1b, RANKL [44-46].

En modulant la réponse immunitaire intestinale, la dysbiose conduit également à une altération de la migration des cellules monocytaires et des lymphocytes vers les tissus, dont la moelle osseuse. Les souris axéniques ont un nombre de monocytes et de précurseurs ostéoclastiques réduit dans la moelle osseuse qui redevient normal après colonisation des animaux avec le microbiote de souris contrôles [28,55]. De plus, les modifications du microbiote altèrent le trafic monocytaire [56]. Dans le contexte de la maladie de Crohn associée à une destruction osseuse sévère et à une dysbiose, les lymphocytes Th17 sont capables de migrer vers la moelle osseuse et d'induire le recrutement de pré-OCLs conduisant à une augmentation très importante de l'ostéoclastogenèse [17]. Contrairement aux OCLs de souris saines qui induisent la génération de lymphocytes $T$ régulateurs, les OCLs générés dans ces conditions inflammatoires activent des lymphocytes T CD4+ producteurs de TNF $\alpha$ [57]. Cette différence est due à l'origine cellulaire des OCLs soulignant le recrutement de progéniteurs ostéoclastiques différents en condition normale et en condition inflammatoire liée à la dysbiose.

\section{5- Futures directions}

De plus en plus d'études soulignent le rôle majeur du microbiote dans la régulation de la masse osseuse pendant la croissance et en condition pathologique. Le microbiote peut agir en combinaison avec d'autres facteurs tels que régime alimentaire, prédisposition génétique, mode de vie, traitement médicamenteux, etc. II peut également agir directement sur certaines réponses par la dissémination des bactéries [58] ou des facteurs qu'elles produisent, entraînant une activation des réactions inflammatoires dans les tissus jusque dans la moelle osseuse. Mais les mécanismes impliqués sont complexes et des études sont encore nécessaires pour les élucider et les contrôler.

La restauration d'un microbiote équilibré est aujourd'hui une approche envisagée pour un certain nombre de ces pathologies. Le microbiote peut être modifié de différentes 
façons, en particulier par le régime alimentaire et la supplémentation en prébiotiques comme les acides gras à chaînes courtes, les oligosaccharides, les carbohydrates et les fibres alimentaires. Ces composés sont métabolisés par certaines souches bactériennes et favorisent donc la croissance de ces souches modifiant ainsi la composition du microbiote. Ce microbiote modifié stimule les réponses anti-inflammatoires et favorise l'absorption intestinale du calcium conduisant à une augmentation de la densité osseuse, comme cela a été démontré par l'administration d'acides gras à chaînes courtes par gavage chez la souris [33]. Le même effet bénéfique sur l'os est rapporté pour les oligosaccharides du lait humain [59]. Les probiotiques ont également un effet notable sur la densité osseuse. Différentes souches de Lactobacillus et Bifidobacterium ont un effet anti-inflammatoire, stimulent l'absorption de la vitamine $\mathrm{D}$ et diminuent la différenciation des OCLs, ce qui explique leur effet protecteur sur la diminution de masse osseuse induite par l'ovariectomie chez la souris [43-45]. L'évaluation de l'effet des probiotiques chez l'homme est en cours dans plusieurs essais cliniques (https://clinicaltrials.gov) mais les résultats ne sont pas encore connus.

Une autre approche est la transplantation de microbiote. Chez la souris, elle est largement utilisée pour démontrer l'implication du microbiote dans de nombreuses conditions pathologiques y compris au niveau osseux $[28,33,46]$. Chez l'homme, la transplantation de microbiote a été testée avec succès dans des pathologies intestinales telles que les colites causées par des infections bactériennes résistantes aux antibiotiques [60]. Cependant, si des études cliniques sont en cours pour évaluer son efficacité dans les maladies rhumatismales, rien n'a été rapporté au niveau osseux. Étant donné l'intérêt grandissant pour la manipulation du microbiote comme approche thérapeutique, des études complémentaires sont encore nécessaires pour mieux comprendre les mécanismes impliqués et évaluer son efficacité dans les pathologies osseuses. Cependant, au vu de leurs effets bénéfiques dans des modèles précliniques, de telles approches ouvrent des perspectives prometteuses pour le traitement des pathologies osseuses.

\section{Remerciements}

Le travail de l'équipe est soutenu par l'ANR (ANR-16-CE14-0030-01), Biocodex, et la Fondation pour la Recherche Médicale (FRM, ECO20160736019). Les figures de cet article ont été réalisées à l'aide de la galerie "Servier Medical Art", http://smart.servier.com/.

\section{Déclaration de liens d'intérêts}

CBW a reçu une subvention de recherche de Biocodex 


\section{6- Références}

[1] Marchesi J, Shanahan F. The normal intestinal microbiota: Curr Opin Infect Dis 2007;20:508-13.

[2] Topping DL, Clifton PM. Short-chain fatty acids and human colonic function: roles of resistant starch and nonstarch polysaccharides. Physiol Rev 2001;81:1031-64.

[3] Ivanov II, Frutos R de L, Manel N, et al. Specific microbiota direct the differentiation of IL-17-producing T-helper cells in the mucosa of the small intestine. Cell Host Microbe 2008;4:337-49.

[4] Gaboriau-Routhiau V, Rakotobe S, Lécuyer E, et al. The key role of segmented filamentous bacteria in the coordinated maturation of gut helper $\mathrm{T}$ cell responses. Immunity 2009;31:677-89.

[5] Hand TW, Vujkovic-Cvijin I, Ridaura VK, Belkaid Y. Linking the Microbiota, Chronic Disease, and the Immune System. Trends Endocrinol Metab TEM 2016;27:831-43.

[6] Van de Wiele T, Van Praet JT, Marzorati M, Drennan MB, Elewaut D. How the microbiota shapes rheumatic diseases. Nat Rev Rheumatol 2016;12:398-411.

[7] Ni J, Wu GD, Albenberg L, Tomov VT. Gut microbiota and IBD: causation or correlation? Nat Rev Gastroenterol Hepatol 2017;14:nrgastro.2017.88.

[8] Kong YY, Yoshida H, Sarosi I, et al. OPGL is a key regulator of osteoclastogenesis, lymphocyte development and lymph-node organogenesis. Nature 1999;397:315-23.

[9] Nakashima T, Hayashi M, Fukunaga T, et al. Evidence for osteocyte regulation of bone homeostasis through RANKL expression. Nat Med 2011;17:1231-4.

[10] Kotake S, Udagawa N, Takahashi N, et al. IL-17 in synovial fluids from patients with rheumatoid arthritis is a potent stimulator of osteoclastogenesis. $J$ Clin Invest 1999;103:1345-52.

[11] Lam J, Takeshita S, Barker JE, Kanagawa O, Ross FP, Teitelbaum SL. TNF-alpha induces osteoclastogenesis by direct stimulation of macrophages exposed to permissive levels of RANK ligand. J Clin Invest 2000;106:1481-8.

[12] Kong Y-Y, Feige U, Sarosi I, et al. Activated $T$ cells regulate bone loss and joint destruction in adjuvant arthritis through osteoprotegerin ligand. Nature 1999;402:304-9.

[13] Wakkach A, Mansour A, Dacquin R, et al. Bone marrow microenvironment controls the in vivo differentiation of murine dendritic cells into osteoclasts. Blood 2008;112:5074-83.

[14] Sato K, Suematsu A, Okamoto K, et al. Th17 functions as an osteoclastogenic helper T cell subset that links T cell activation and bone destruction. J Exp Med 2006;203:2673-82.

[15] Pöllinger B, Junt T, Metzler B, et al. Th17 cells, not IL-17+ y $\delta T$ cells, drive arthritic bone destruction in mice and humans. J Immunol Baltim Md 1950 2011;186:2602-12.

[16] Oostlander AE, Everts V, Schoenmaker T, et al. T cell-mediated increased osteoclast formation from peripheral blood as a mechanism for Crohn's disease-associated bone loss. J 
Cell Biochem 2012;113:260-8.

[17] Ciucci T, Ibáñez L, Boucoiran A, et al. Bone marrow Th17 TNFa cells induce osteoclast differentiation, and link bone destruction to IBD. Gut 2015;64:1072-81.

[18] Peters A, Lee Y, Kuchroo VK. The many faces of Th17 cells. Curr Opin Immunol $2011 ; 23: 702-6$.

[19] Ivanov II, Atarashi K, Manel N, et al. Induction of intestinal Th17 cells by segmented filamentous bacteria. Cell 2009;139:485-98.

[20] Khor B, Gardet A, Xavier RJ. Genetics and pathogenesis of inflammatory bowel disease. Nature 2011;474:307-17.

[21] Wu H-J, Ivanov II, Darce J, et al. Gut-residing segmented filamentous bacteria drive autoimmune arthritis via T helper 17 cells. Immunity 2010;32:815-27.

[22] Glatigny S, Fert I, Blaton MA, et al. Proinflammatory Th17 cells are expanded and induced by dendritic cells in spondylarthritis-prone HLA-B27-transgenic rats. Arthritis Rheum 2012;64:110-20.

[23] Nograles KE, Zaba LC, Guttman E, et al. Th17 cytokines interleukin (IL)-17 and IL-22 modulate distinct inflammatory and keratinocyte-response pathways. $\mathrm{Br} \mathrm{J}$ Dermatol 2008;159:1092-102.

[24] Stotzer P-O, Johansson C, Mellström D, Lindstedt G, Kilander AF. Bone mineral density in patients with small intestinal bacterial overgrowth. Hepatogastroenterology 2003;50:1415-8.

[25] Wallace JG, Gohir W, Sloboda DM. The impact of early life gut colonization on metabolic and obesogenic outcomes: what have animal models shown us? J Dev Orig Health Dis 2016;7:15-24.

[26] Clarke G, Stilling RM, Kennedy PJ, Stanton C, Cryan JF, Dinan TG. Minireview: Gut microbiota: the neglected endocrine organ. Mol Endocrinol Baltim Md 2014;28:1221-38.

[27] Kennedy PJ, Cryan JF, Dinan TG, Clarke G. Kynurenine pathway metabolism and the microbiota-gut-brain axis. Neuropharmacology 2017;112:399-412.

[28] Sjögren K, Engdahl C, Henning $P$, et al. The gut microbiota regulates bone mass in mice. J Bone Miner Res Off J Am Soc Bone Miner Res 2012;27:1357-67.

[29] Ohlsson C, Nigro G, Boneca IG, Bäckhed F, Sansonetti P, Sjögren K. Regulation of bone mass by the gut microbiota is dependent on NOD1 and NOD2 signaling. Cell Immunol 2017;317:55-8.

[30] Schwarzer M, Makki K, Storelli G, et al. Lactobacillus plantarum strain maintains growth of infant mice during chronic undernutrition. Science 2016;351:854-7.

[31] Fransen F, Zagato E, Mazzini E, et al. BALB/C and C57BL/6 Mice Differ in Polyreactive IgA Abundance, which Impacts the Generation of Antigen-Specific IgA and Microbiota Diversity. Immunity 2015;43:527-40. 
[32] Stanisavljević S, Đedović N, Vujičić M, et al. Strain-specific helper T cell profile in the gut-associated lymphoid tissue. Immunol Lett 2017;190:282-8.

[33] Yan J, Herzog JW, Tsang K, et al. Gut microbiota induce IGF-1 and promote bone formation and growth. Proc Natl Acad Sci U S A 2016;113:E7554-63.

[34] Yakar S, Courtland H-W, Clemmons D. IGF-1 and bone: New discoveries from mouse models. J Bone Miner Res Off J Am Soc Bone Miner Res 2010;25:2543-52.

[35] Cox LM, Yamanishi S, Sohn J, et al. Altering the intestinal microbiota during a critical developmental window has lasting metabolic consequences. Cell 2014;158:705.

[36] Yurkovetskiy L, Burrows M, Khan AA, et al. Gender bias in autoimmunity is influenced by microbiota. Immunity 2013;39:400-12.

[37] Mueller S, Saunier K, Hanisch C, et al. Differences in fecal microbiota in different European study populations in relation to age, gender, and country: a cross-sectional study. Appl Environ Microbiol 2006;72:1027-33.

[38] Dominianni C, Sinha R, Goedert JJ, et al. Sex, body mass index, and dietary fiber intake influence the human gut microbiome. PloS One 2015;10:e0124599.

[39] Baker JM, Al-Nakkash L, Herbst-Kralovetz MM. Estrogen-gut microbiome axis: Physiological and clinical implications. Maturitas 2017;103:45-53.

[40] Roggia C, Gao Y, Cenci S, et al. Up-regulation of TNF-producing T cells in the bone marrow: A key mechanism by which estrogen deficiency induces bone loss in vivo. Proc Natl Acad Sci 2001;98:13960-5.

[41] Cenci S, Weitzmann MN, Roggia C, et al. Estrogen deficiency induces bone loss by enhancing T-cell production of TNF- $\alpha$. J Clin Invest 2000;106:1229-37.

[42] D'Amelio P, Grimaldi A, Di Bella S, et al. Estrogen deficiency increases osteoclastogenesis up-regulating $\mathrm{T}$ cells activity: a key mechanism in osteoporosis. Bone 2008;43:92-100.

[43] Parvaneh K, Ebrahimi M, Sabran MR, et al. Probiotics ( Bifidobacterium longum ) Increase Bone Mass Density and Upregulate Sparc and Bmp-2 Genes in Rats with Bone Loss Resulting from Ovariectomy. BioMed Res Int 2015;2015:1-10.

[44] Britton RA, Irwin R, Quach D, et al. Probiotic L. reuteri Treatment Prevents Bone Loss in a Menopausal Ovariectomized Mouse Model. J Cell Physiol 2014;229:1822-30.

[45] Ohlsson C, Engdahl C, Fåk F, et al. Probiotics protect mice from ovariectomy-induced cortical bone loss. PloS One 2014;9:e92368.

[46] Li J-Y, Chassaing B, Tyagi AM, et al. Sex steroid deficiency-associated bone loss is microbiota dependent and prevented by probiotics. J Clin Invest 2016;126:2049-63.

[47] Wang J, Wang Y, Gao W, et al. Diversity analysis of gut microbiota in osteoporosis and osteopenia patients. PeerJ 2017;5:e3450.

[48] Taurog JD, Richardson JA, Croft JT, et al. The germfree state prevents development 
of gut and joint inflammatory disease in HLA-B27 transgenic rats. $J$ Exp Med 1994;180:2359-64.

[49] Vaahtovuo J, Munukka E, Korkeamäki M, Luukkainen R, Toivanen P. Fecal microbiota in early rheumatoid arthritis. J Rheumatol 2008;35:1500-5.

[50] Zhang $\mathrm{X}$, Zhang $\mathrm{D}$, Jia $\mathrm{H}$, et al. The oral and gut microbiomes are perturbed in rheumatoid arthritis and partly normalized after treatment. Nat Med 2015;21:895-905.

[51] Breban M, Tap J, Leboime A, et al. Faecal microbiota study reveals specific dysbiosis in spondyloarthritis. Ann Rheum Dis 2017;76:1614-22.

[52] Amdekar S, Singh V, Singh R, Sharma P, Keshav P, Kumar A. Lactobacillus casei reduces the inflammatory joint damage associated with collagen-induced arthritis (CIA) by reducing the pro-inflammatory cytokines: Lactobacillus casei: COX-2 inhibitor. J Clin Immunol 2011;31:147-54.

[53] Kerr SW, Wolyniec WW, Filipovic Z, et al. Repeated measurement of intestinal permeability as an assessment of colitis severity in HLA-B27 transgenic rats. J Pharmacol Exp Ther 1999;291:903-10.

[54] Martínez-González O, Cantero-Hinojosa J, Paule-Sastre P, Gómez-Magán JC, Salvatierra-Ríos D. Intestinal permeability in patients with ankylosing spondylitis and their healthy relatives. Br J Rheumatol 1994;33:644-7.

[55] Khosravi A, Yáñez A, Price JG, et al. Gut Microbiota Promote Hematopoiesis to Control Bacterial Infection. Cell Host Microbe 2014;15:374-81.

[56] Bain CC, Bravo-Blas A, Scott CL, et al. Constant replenishment from circulating monocytes maintains the macrophage pool in the intestine of adult mice. Nat Immunol 2014;15:929-37.

[57] Ibáñez L, Abou-Ezzi G, Ciucci T, et al. Inflammatory osteoclasts prime TNFaproducing CD4(+) T cells and express CX3 CR1. J Bone Miner Res 2016;31:1899-1908.

[58] Burcelin R, Serino M, Chabo C, et al. Metagenome and metabolism: the tissue microbiota hypothesis. Diabetes Obes Metab 2013;15:61-70.

[59] Charbonneau MR, O'Donnell D, Blanton LV, et al. Sialylated Milk Oligosaccharides Promote Microbiota-Dependent Growth in Models of Infant Undernutrition. Cell 2016;164:859-71.

[60] Cammarota G, laniro G, Gasbarrini A. Fecal Microbiota Transplantation for the Treatment of Clostridium difficile Infection: A Systematic Review. J Clin Gastroenterol 2014;48:693-702. 


\section{Légendes des figures}

Figure 1 : Lien entre microbiote et tissu osseux.

Le microbiote intestinal participe au maintien de l'intégrité de la barrière intestinale et à la maturité du système digestif. Les modifications du microbiote entraînent une dissémination accrue des composants dérivés des bactéries, et des changements d'expression de cytokines et facteurs de croissance. Cela conduit à des altérations de la réponse des systèmes immunitaire, endocrinien vasculaires et nerveux qui sont tous des régulateurs de la différenciation et/ou fonction des cellules osseuses.

Figure 2 : Modifications du microbiote et différenciation des ostéoclastes.

Les modifications du microbiote induisent une stimulation des lymphocytes Th17 et une augmentation de la production des cytokines ostéoclastogéniques TNF $\alpha$, IL-17 et RANKL au niveau intestinal. Les lymphocytes Th17 migrent vers la moelle osseuse et l'augmentation des mêmes cytokines inflammatoires dans ce tissu entraîne une différenciation accrue des ostéoclastes conduisant à une baisse de la densité osseuse. 

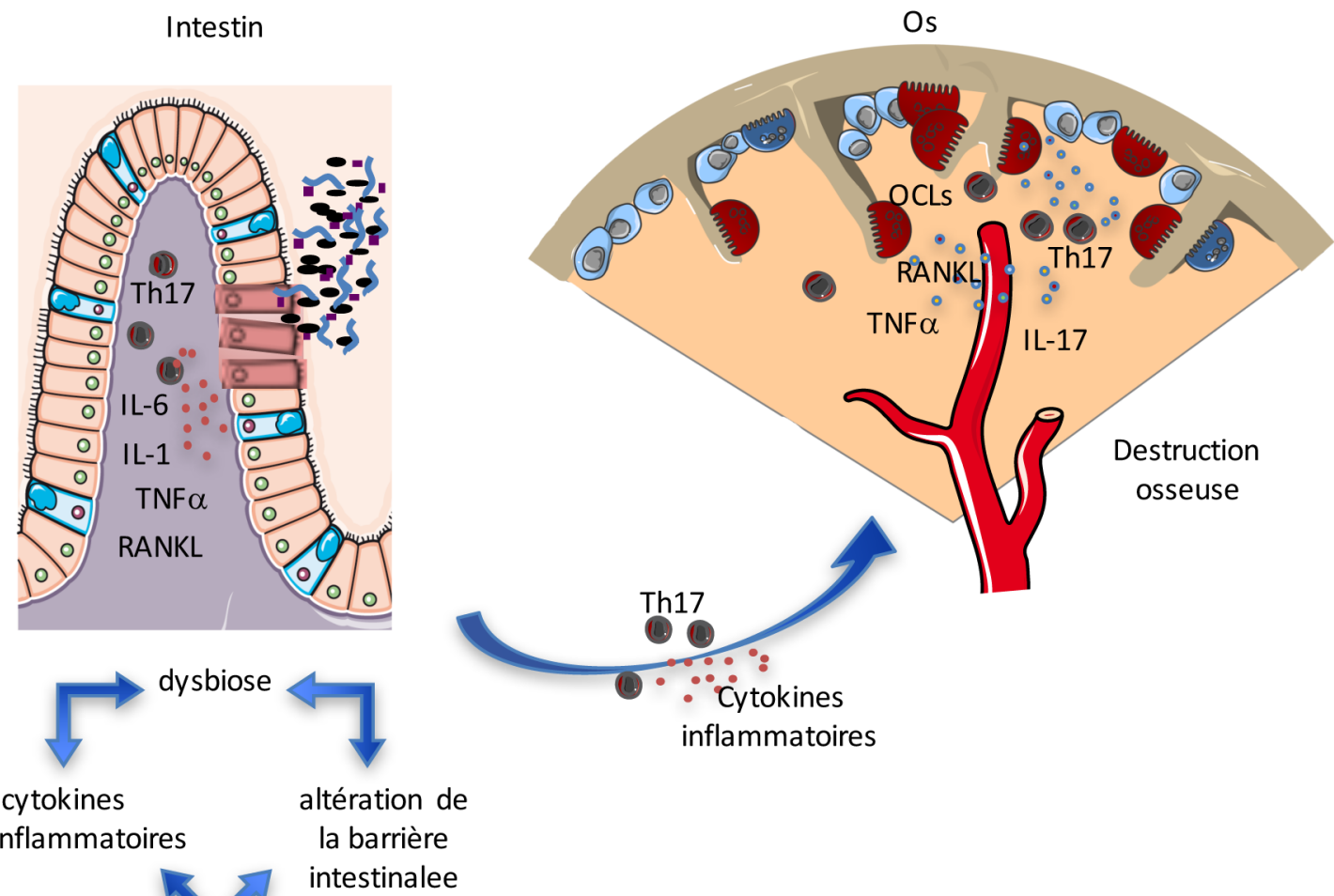